\title{
An Alternative Description of the Schwarzschild Black Hole
}

\author{
Yuriy Zayko \\ Division of Applied Informatics, Russian Presidential Academy of National Economy and Public Administration, \\ Stolypin Volga Region Institute, Saratov, Russia \\ Email: zyrnick@rambler.ru
}

\begin{abstract}
The article gives the metric of the Schwarzschild black hole, consistent with the fact that the electromagnetic vacuum is unstable under its horizon. A similar metric was previously obtained in the author's work when studying the dynamics of a vortex. This metric is characterized by the absence of many familiar concepts intrinsic to hyperbolic space-time: light-like geodesics, light cone, etc. The thermodynamic aspect of this black hole model is also considered.
\end{abstract}

Keywords: Metric, vortex, electromagnetic vacuum, black hole.

\section{Introduction}

The Schwarzschild solution of the Einstein equations plays an important role in applications. As noted in [1]: "In fact, the basic observational data confirming the general theory of relativity are based on the predictions obtained for this solution." Briefly list the basic information about it.

The Schwarzschild metric in the coordinates $\left(x^{0}, x^{1}, x^{2}, x^{3}\right)=(c t, r, \theta, \phi)$ looks as follows

$$
d s^{2}=\left(1-\frac{2 M}{r}\right)(c d t)^{2}-\left(1-\frac{2 M}{r}\right)^{-1} d r^{2}-r^{2}\left(d \theta^{2}+\sin ^{2} \theta d \phi^{2}\right)
$$

where $M$ has physical meaning of the mass of the particle ${ }^{1}$, which creates the gravitational field. For $r>r_{g}=2 M / c^{2}$ coordinate $r$ coincides with the radial coordinate in the spherical coordinate system of a three-dimensional Euclidean space, and the coordinate $t$ plays the role of time. Value $r_{g}$ is called the gravitational radius, and the sphere of radius $r_{q}$ determines the so-called event horizon [2]. For $0<r<r_{g} t$ and $r$ lose the sense of temporal and spatial coordinates. Since this area is directly related to the physics of the black hole, the description of the latter depends on the sense in which these coordinates are considered here. It should be clearly stated that at present time there are no real grounds for carrying out this procedure; therefore, the decision is made on the basis of other considerations.

It is commonly believed that in the region below the horizon the coordinates $t$ and $r$ are changing one another: $t$ becomes a space-like coordinate, and $r$ - time-like [3]. The meaning of this redefinition is dictated by the expression for the interval (1): as a result, the interval retains its form, and the description of the sub-horizontal region can be performed in the same terms as the region above the horizon, using the notions of light-like geodesics, light cone, etc. This is due to the preservation of the hyperbolic nature of space-time, which allows us to talk about the propagation of signals in it in accordance with the usual notions of causality. In other words, the choice of such a scenario is conditioned by the desire to obtain a description of the sub-horizontal region as close as possible to the description of the region above the horizon. The main role in it, as in the region above the horizon, is played by light-like geodesics, as the world lines of photons. This, in turn, makes it possible to smoothly sew along the horizon the metrics above and below it.

Note that these results do not in any way use Maxwell's equations; in particular, the question of the stability of the electromagnetic vacuum under the horizon is not investigated. The problem arises from the fact that, as shown in [4], it is here that the electromagnetic vacuum loses stability being stable over the horizon. The consequence of this is the impossibility of the existence of light waves, i.e. photons

${ }^{1}$ For our purposes it suffices to consider a point particle 
under the horizon. For this reason, the above redefinition of coordinates $t$ and $r$ and with this the traditional picture of the physics of the black hole lose its meaning. It is required to look for other ways of describing it, consistent with the discovered facts. Below is an example of a metric under the horizon in which there are no light-like geodesics.

\section{The Metric of a Black Hole}

Let us apply the approach previously used in [5] for the description of a vortex to the description of the Schwarzschild black hole (SBH) metric. In the equatorial plane $\theta=\pi / 2$ the metric of the Schwarzschild problem (1) can be rewritten in the form

$$
\begin{aligned}
& d s^{2}=A(r)(c d t)^{2}-B(r) d r^{2}-r^{2} d \phi^{2} \\
& A(r)=1-\frac{r_{g}}{r}, B(r)=\left(1-\frac{r_{g}}{r}\right)^{-1}
\end{aligned}
$$

Write the equation of motion for massive particle $\frac{d^{2} x^{i}}{d s^{2}}+\Gamma_{k l}^{i} \frac{d x^{k}}{d s} \frac{d x^{l}}{d s}=0$, where $\Gamma_{k l}^{i}$ - are the Christoffel symbols, corresponding (2) [2]. Details of the calculations can be found, for example, in [2]. We immediately write down the result $[2]$

$$
\begin{aligned}
& \frac{d \phi}{d x}=\frac{\rho}{y^{2}} \\
& \left(\frac{d y}{d x}\right)^{2}=1-\frac{1}{e^{2}}\left(1-\frac{1}{y}\right)-\mu^{2}\left(1-\frac{1}{y}\right) \frac{1}{y^{2}} \\
& y=\frac{r}{r_{g}}, x=\frac{s}{r_{g}}, e=\frac{E}{m c^{2}}, \mu=\frac{\rho}{r_{g}}, \rho=\frac{K c}{E}
\end{aligned}
$$

where $E$ and $K$ are the total energy and angular momentum, $\rho$ is the impact distance, and $m$ is the mass of the moving particle. These equations have been thoroughly investigated $[2,3]$.

The metric (2) so as (1) cannot be used below the horizon. For this purpose following [2], we could use the Finkelstein transformation $r, t \rightarrow R, \tau$ (signs \pm correspond to the motion to or out of center $r=$ $0)$

$$
d r=\frac{f(d R \pm c d \tau)}{\sqrt{A B}}, c d t=\frac{f^{2} d R \pm c d \tau}{A}
$$

where $f$ - is a function chosen from the condition that the fictitious singularity at $r=r_{g}$ of the metrics (1) and (2) be eliminated. The final expression for $f(r)$ looks as follows

$$
f(r)=[1-A(r)]^{1 / 2}=\sqrt{\frac{r_{g}}{r(R, \tau)}}
$$

The resulting metric in our notations coincides with the famous one [2] and looks as follows

$$
d s^{2}=(c d \tau)^{2}-f^{2}(r) d R^{2}-r^{2}(R, \tau) d \phi^{2}
$$

But this way as was noted in [5] leads to the contradiction which consists in that from the one hand we receive the synchronous reference system and from the other, the observers located in the different sides of the horizon cannot exchange the light signals. Therefore, we can use the transformation (4) only above the horizon. This indicates the incompleteness of the Finkelstein coordinates [2]. This difficulty cannot be overcome with the transition to coordinate system like Kruskal-Szekeres for example which is more complete than Finkelstein system. All of those coordinate systems do not take into account the fact of instability of electromagnetic vacuum below the horizon of the black hole. Thus, following [5] we use coordinate transformations which differ from (4) by the absence of new time $\boldsymbol{T}$, other words we use under horizon the metric 


$$
\begin{aligned}
& d s^{2}=-\frac{r_{g}}{r(R)} d R^{2}-r^{2}(R) d \phi^{2} \\
& R=\int \sqrt{\frac{A B}{1-A}} d r=\frac{2}{3} \sqrt{\frac{r^{3}}{r_{g}}}
\end{aligned}
$$

This metric has no light geodesic what is consistent with absence of photons below the horizon. The equations of motion corresponding to this metric look as follows

$$
\begin{aligned}
& \frac{d^{2} \phi}{d s^{2}}+2 \frac{1}{r(R)} \frac{d r}{d s} \frac{d \phi}{d s}=0 \\
& \frac{d^{2} R}{d s^{2}}-\frac{1}{2 r} \frac{d r}{d R}\left(\frac{d R}{d s}\right)^{2}-\frac{r^{2}}{r_{g}} \frac{d r}{d R}\left(\frac{d \phi}{d s}\right)^{2}=0
\end{aligned}
$$

After a single integration of the first of these equations, we obtain the previous result (see the first equation (3)). Integrating the second equation once, and determining the integration constant from the cross-linking condition with the solution of (3), we obtain in dimensionless form

$$
\left(\frac{d y}{d x}\right)^{2}=1+\mu^{2}\left(1-\frac{1}{y^{2}}\right)
$$

where $\boldsymbol{\mu}$ - is the dimensionless angular momentum (3). The presence of this integral is a consequence of the isotropy of space that is conserved in our model. Another feature of the proposed model is the violation of invariance with respect to shifts in time under the horizon of the black hole, which is expressed in the absence of an energy integral in (9), in contrast to (3).

Equations (3) and (9) give a description of the motion, respectively, above and below the horizon, of the test particle in the black hole field in our model. The Figures 1,2 show the trajectories of their solutions on the phase plane for different values of $e$ and $\mu$.
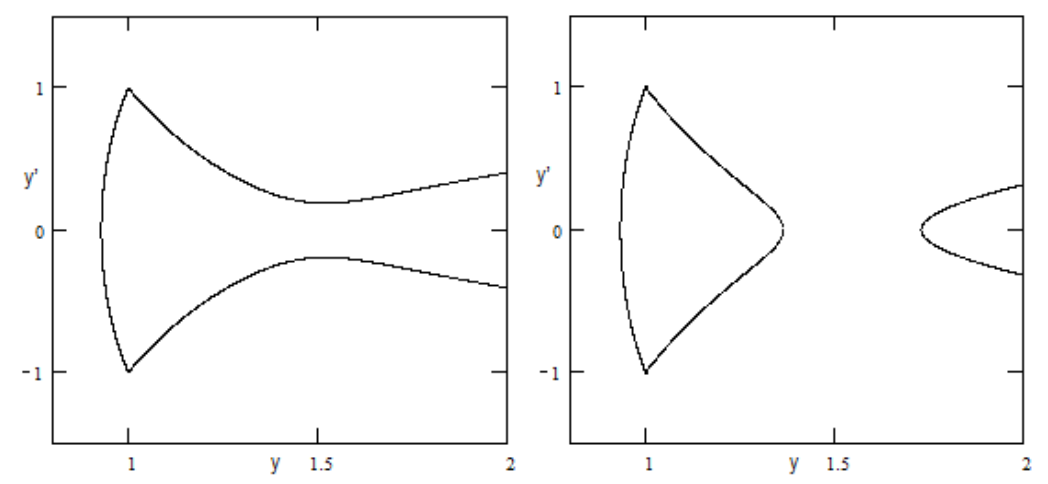

Figure 1. The phase portrait of the solutions of equations (3) and (9) for $e=3, \boldsymbol{\mu}=2,5$ (left) and $e=3, \boldsymbol{\mu}=2,6$ (right)

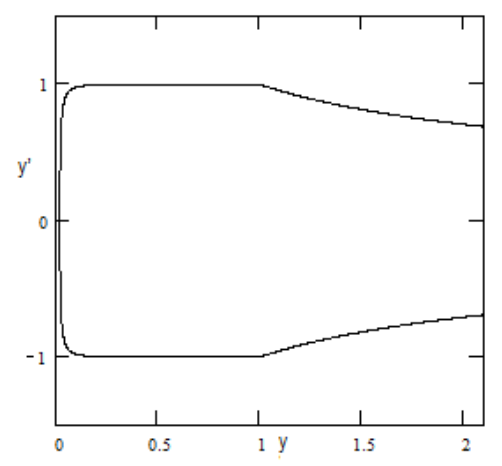

Figure 2. The phase portrait of the solutions of equations (3) and (9) for $e=1, \boldsymbol{\mu}=0,02$

It follows from the calculations that the nature of the solution depends to a large extent on the 
magnitude of the angular momentum of the particle $\boldsymbol{\mu}$. For small values of $\boldsymbol{\mu}$, when the radicand in (3) has two complex conjugate roots, the outer part of the phase trajectory above the horizon $(y>1)$ consists of one branch describing the infinite motion. (Fig.1, left). With increasing $\mu$, when the radicand in (3) has two different real roots, the outer part of the phase trajectory consists of two branches. One of them corresponds to an infinite motion of a particle without capture by a black hole. The other, together with the inner part of the phase trajectory below the horizon, describes the finite, periodic motion of a particle in the field of a black hole without falling to the center $\left(y_{0}<y<y_{1}, y_{0}=\mu / \sqrt{1+\mu^{2}}, y_{0}\right.$-is the branch point of the inner part of the trajectory, $y_{1}$ is the smallest branch point of the outer part of the trajectory) (Fig.1, right). On the horizon $(y=1)$ inner and outer parts of the trajectory are sewn together. As $\mu$ decreases to zero, the outer part of the trajectory consists of one branch, and the inner part approaches to the center of the field $r=0$ infinitely close.

As can be seen from the solutions obtained, the particle, falling into a black hole after crossing the horizon, is able to return back.

\section{Thermodynamics of a Black Hole.}

In this section, we show that in a black hole there is a loss of information that falls under the horizon along with the material carrier. To do this, we investigate the behavior of the black hole in our model from the point of view of thermodynamics.

Most of the results concerning the thermodynamics of black holes in frame of traditional description are based on the so-called second law of the dynamics of a black holes, which states that for any processes the surface area of the black hole $S_{\mathrm{BH}}$ does not decrease $[3,6]$. If we use the analogy between $\mathrm{S}_{\mathrm{BH}}$ and entropy, then the last assertion is equivalent to the second law of thermodynamics when applied to the physics of black holes. Since its proof is based on argumentation that uses all the attributes of the traditional theory of black holes and which we cannot use, another approach to the problem is presented below.

The covariant expression for the second law of thermodynamics has the form [7]

$$
\frac{\partial}{\partial x^{v}}\left(\phi_{0} \frac{d x^{v}}{d s}\right) \delta x^{0} \delta x^{1} \delta x^{2} \delta x^{3} \geq \frac{\delta Q_{0}}{T_{0}}
$$

where $x^{v}$ - are four-dimensional coordinates $(v=0,1,2,3), \frac{d x^{v}}{d s}-$ four-speed, $\phi_{0}$ - entropy density, $\delta Q_{0}$ - the amount of heat entering the element of a four-dimensional volume $\delta x^{0} \delta x^{1} \delta x^{2} \delta x^{3}$ at the temperature $T_{0}$. The subscript 0 refers to the observer's own reference frame.

We apply (10) to the black hole in our model. Then the spatial volume $\delta x^{1} \delta x^{2} \delta x^{3}$ represents the volume of SBH bounded by the surface of the horizon. According to the above $\delta x^{0}=0$. This allows us to rewrite $(10)$ in the form

$$
\frac{\delta Q_{0}}{T_{0}} \leq 0
$$

If, as usual $T_{0} \geq 0$, then we conclude that the black hole in our model can only give heat to its environment, what is a consequence of the elimination of information in accordance with the Landauer principle [8]. Our analysis, however, leaves open the question of whether all the information that has got under the horizon was destroyed there.

\section{Discussion}

The results obtained in the paper disagree with the traditional representation of the SBH, both about the geometry of a black hole, and about the motion in its field [2]. There are no traditional elements of description: signals, light-like geodetic, light cone, absolute future, and past, etc. All this is a consequence of the instability of the electromagnetic vacuum and, accordingly, the absence of photons under the horizon.

Space-time under the horizon is not hyperbolic; moreover, its description does not use time. In general, 
this description differs from the traditional Newtonian and is closer to the description suggested by Leibniz, according to which the characteristics of objects are not their immanent properties, but are determined by their interaction with the environment. In particular "Time must be a consequence of change; without alteration in the world, there can be no time" [9].

The absence of light-like geodesics beneath the horizon does not allow smooth sewing of metrics along the horizon, which makes it impossible to construct diagrams of the Carter-Penrose type [1]. Instead, a partial cross-linking of particle trajectories on the horizon using the equations of motion is performed. As a result, on the trajectory, the second derivative $d^{2} r / d s^{2}$ has a break at the horizon, which means the appearance of an additional force to already known forces and can serve as a factor to judge the truth of the given model.

From the foregoing it follows that the loss of information in a black hole, accompanied by a flow of heat from it to the environment, is not necessarily related to the loss of matter under the horizon. This is in agreement with the assertion of N. Wiener "Information is information, not matter or energy. No materialism which does not admit this can survive at the present day" [10]. It should be said that the currently accepted theory of black holes ignores this assertion [3, 6].

Summarizing, we can say that with the assumptions made about the instability of the electromagnetic vacuum under the horizon of the Schwarzschild black hole, the proposed alternative description of it is well grounded from the philosophical point of view. To give it the features of a physical theory, it will take time and efforts of specialists, at least comparable to the efforts have spent on building the traditional theory of black holes.

\section{Conclusion}

The article investigates the metric of the Schwarzschild black hole (SBH), which agrees with the fact of the absence of photons under its horizon, which is caused by the instability of the electromagnetic vacuum in this region [4]. This geometry differs significantly from the traditional hyperbolic geometry, characterized only by a rearrangement of the spatial and temporal coordinates under the horizon of the SBH. This approach does not allow smooth matching of metrics on both sides of the black hole horizon. Various trajectories allowed by this metric are investigated. The thermodynamic aspect of this black hole model is also considered.

Earlier the metric of this type allowed us to calculate the Riemann $\zeta$-function in the complex plane and to prove the Riemann hypothesis [5]. The proof used some elements of the general theory of relativity, in particular, a description of the dynamics of a material particle in a medium having a horizon, analogous to the horizon of a black hole. Therefore, it is of independent interest to investigate the Schwarzschild black hole under the same assumption as in [5].

\section{References}

1. M.O. Katanaev, Geometric methods in mathematical physics, arXiv: 1311.0733v3 [math-ph] 20 Nov 2016.

2. L.D. Landau, E.M. Lifshitz, The Classical Theory of Fields, (4th ed.), Butterworth-Heinemann, 1975.

3. C.W. Misner, K.S. Thorne, J.A. Wheeler, Gravitation, in 3 vol., W.H. Freeman and Co, San Francisco, 1973.

4. Y.N. Zayko, "Maxwel's Electrodynamics in Curved Space-Time," World Journal of Innovative Research, vol. 1, no. 1, pp. 16-19, 2016,

5. Y.N. Zayko, "The Proof of the Riemann Hypothesis on a Relativistic Turing Machine," International Journal of Theoretical and Applied Mathematics, vol. 3, no. 6, pp. 219-224, 2017.

6. J. D. Bekenstein, "Black Holes and Entropy," Phys. Rev. D, vol. 7, no. 8, pp. 2333-2346, 1973.

7. R.C. Tolman, Relativity, Thermodynamics and Cosmology. Oxford, at the Clarendon Press, 1969.

8. R. Landauer, "Irreversibility and Heat Generation in the Computing Process," IBM Journ. Res. Develop., vol. 5, pp. $183-191,1961$.

9. L. Smolin, Time Reborn: From the Crisis in Physics to the Future of the Universe, Houghton Mifflin Harcourt, 2013.

10. N. Wiener, Cybernetics: Or Control and Communication in the Animal and the Machine, Paris, (Hermann \& Cie) \& Camb. Mass. (MIT Press) ISBN 978-0-262-73009-9; 1948, 2nd revised ed. 1961. 